\title{
Histamine challenge testing: comparison of three methods
}

\author{
JOHN BRITTON, AHMED MORTAGY, ANNE TATTERSFIELD \\ From Basingstoke General Hospital, Basingstoke, and Southampton General Hospital, Southampton
}

ABSTRACT Non-specific bronchial reactivity is related to the severity of clinical asthma. Histamine \pm challenge testing is increasingly used in association with questionnaires in epidemiological studies of $i$ the prevalence and morbidity of asthma in the community. The histamine challenge method $\vec{N}$ described by Cockcroft $e t$ al is widely used and well standardised but it has disadvantages for ${ }^{\infty}$

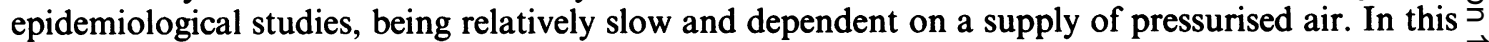
study we have assessed two simpler methods, one described by Yan et al and one by Mortagy, and $\rightarrow$ compared these with the Cockcroft method. Twenty four adults with asthma were tested with each $\frac{\mathbb{D}}{\square}$ method in set order in a balanced design, and retested with each method in the same order. The $\frac{2}{9}$ Mortagy and Yan methods recorded $\mathrm{PC}_{20}$ or $\mathrm{PD}_{20}$ values on both occasions in all 24 subjects, but $₹$ the Cockcroft method only 16 subjects. Repeatability, assessed as the $95 \%$ range for a single $\vec{\oplus}$ measurement, did not differ significantly, being $\pm 1.94,2.11$, and 2.40 doubling concentrations for ${ }^{\infty}$ the Mortagy, Yan, and Cockcroft methods respectively. The Mortagy and Yan methods required less cumbersome equipment and took under 15 minutes to complete, compared with up to $45 \sum_{3}$ minutes for the Cockcroft technique. The similar repeatability of all three methods in these subjects suggests that the two faster techniques are viable alternatives. The technical problems of standardising the Mortagy method lead us to conclude that, of the three methods compared, the Yan $\stackrel{\Phi}{\varrho}$ technique offers the greatest advantages for epidemiological studies.

Since Tiffeneau introduced the concept of an inhalation challenge with histamine as a diagnostic test for asthma in $1958,{ }^{1}$ many different methods of testing have been developed. Of these, the method described by Cockcroft et $a l^{2}$ using the Wright nebuliser is particularly well standardised and has been widely adopted in clinical and research work.

The measurement of non-specific reactivity is of great potential value in the epidemiological investigation of asthma, but in this application the Cockcroft method has certain disadvantages. The test is slow, taking up to $\mathbf{4 5}$ minutes to complete, and it is dependent on a supply of pressurised air, making the equipment bulky and relatively expensive.

Recently two alternative methods have been used for epidemiological studies. Yan et $^{\mathrm{a} l^{3}}$ have described a method using the hand held DeVilbiss No 40 nebuliser and Mortagy ${ }^{4}$ a method based on the Pul-

Address for reprint requests: Dr John Britton, Respiratory Medicine Unit, City Hospital, Nottingham NG5 IPB.

Accepted 9 September 1985 masonic ultrasonic nebuliser. Both are completed more quickly than the Cockcroft method, and neither requires an air supply, but their repeatability is less음 well documented than that of the Cockcroft tech- $\frac{\hat{\otimes}}{x}$ nique.

In order to select the most suitable method for an epidemiological survey we have compared mea-o surements of histamine reactivity using all three methods (Cockcroft, Yan, and Mortagy) in a group of 0 asthmatic subjects, assessing the methods for repeat- $\rightarrow$ ability and comparability of response.

\section{Methods}

Twenty four subjects with asthma were recruited $\stackrel{N}{\mathcal{N}}$ from outpatient clinics and hospital staff in Basing- 0 stoke and Southampton. Criteria for inclusion were: 0 age 18-55 years, mild asthma requiring $\beta$ agonist $\underset{\mathbb{D}}{\stackrel{C}{(}}$ treatment in the previous 12 months but with stable $\stackrel{\odot}{+}$ medication requirements in the last six weeks, and a 0 resting $\mathrm{FEV}_{1}$ above $60 \%$ of the predicted value. ${ }^{5}$

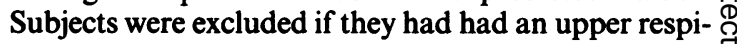
ratory tract infection in the previous six weeks, or if $\frac{\mathbb{Q}}{\mathbb{Q}}$ 
they were taking oral steroids, antihistamines, sodium cromoglycate, ipratropium bromide, or theophylline derivatives, but not if they were taking inhaled steroids (continued as usual throughout the study), or inhaled $\beta$ agonists (withheld for six hours before the study). Each subject was tested once with each of the tisree methods and these were then repeated in the same order, so that there was a total of six tests. The order of tests was determined from a balanced design in which all orders were represented equally.

All tests were performed at the same time of day for each subject and at least one but not more than seven days apart. Tests were performed only if baseline FEV 1 was within $10 \%$ of mean preceding baseline values. If at the end of six studies any one baseline FEV $_{1}$ value was outside $10 \%$ of the collective mean, that study was repeated. The study was approved by the Basingstoke and Southampton ethical committees and was carried out in the winter months of 1983-4.

The method protocols were taken from published accounts by their designers. ${ }^{2-4}$ Details of the individual methods are summarised below. For all methods, solutions of histamine (histamine acid phosphate, BDH Chemicals, Poole) were made up to the appropriate concentrations in normal saline on the day of the test.

All tests were administered with the subject seated. Successive concentrations or doses of histamine were given until the dose regimen was complete or the subject's $\mathrm{FEV}_{1}$ had fallen to below $75 \%$ of the postsaline value. At the end of the test $200 \mu \mathrm{g}$ salbutamol was given by metered dose aerosol if the $\mathrm{FEV}_{1}$ had fallen by $10 \%$ or more, or if the subject felt wheezy or tight in the chest. The subject was then supervised until the $\mathrm{FEV}_{1}$ had returned to pretest levels.

\section{THE THREE METHODS}

\section{Cockcroft method ${ }^{2}$}

A Wright nebuliser was driven by air at a flow of 81 $\min ^{-1}$, which was known from preliminary studies to produce an output of $0.14 \mathrm{ml} \mathrm{min} \mathrm{m}^{-1}$. It was primed with $3 \mathrm{ml}$ solution, and the aerosol was delivered directly to the subject via a face mask without intervening tubing. The subject, wearing a noseclip, inhaled the aerosol by breathing tidally for two minutes with the face mask held loosely over the mouth and nose.

Baseline $\mathrm{FEV}_{1}$ and forced vital capacity (FVC) measurements were recorded until two successive measurements were within 5\%. After each inhalation, including that of normal saline, $\mathrm{FEV}_{1}$ was recorded once, unless technically unsatisfactory, at 30 and 90 seconds and at subsequent 90 second intervals until the value was higher than the previous reading. The lowest postinhalation value was taken for analysis. After baseline spirometric measurements the subject inhaled saline, followed by successive inhalations of histamine in doubling concentrations from 0.3 to $8 \mathrm{mg} / \mathrm{ml}$.

\section{Yan method ${ }^{3}$}

The output of several DeVilbiss No 40 nebulisers was measured to select five with an output as close as possible to $0.003 \mathrm{ml}$ per puff, and within the authors' specified limits of $0.0018-0.0042 \mathrm{ml}$. These were primed with $1 \mathrm{ml}$ of either saline or histamine in concentrations of $0.3,0.6,2.5$, or $5 \mathrm{~g} / 100 \mathrm{ml}$.

Baseline $\mathrm{FEV}_{1}$ was measured, the greater of two consecutive measurements within $200 \mathrm{ml}$ being taken. Inhalations were administered by expressing one or more puffs from the nebuliser directly in front of the subject's open mouth, at the beginning of a near maximal inspiration from functional residual capacity. The inspiration was held for three seconds.

Three puffs of saline were given in successive inhalations. Measurement of $\mathrm{FEV}_{1}$ was repeated one minute after the last inhalation, and followed immediately by the first inhalation of histamine. Histamine was administered according to the regimen shown in the table, to achieve cumulative doubling doses of 0.03-7.8 $\mu \mathrm{mol}$.

\section{Mortagy method ${ }^{4}$}

A Pulmasonic ultrasonic nebuliser, primed with $3 \mathrm{ml}$ of solution, generated aerosol continuously. The subject breathed tidally through the nebuliser mouthpiece, and the mouthpiece valve system ensured that aerosol was emitted only during inspiration. Baseline $\mathrm{FEV}_{1}$ and FVC were recorded until three $\mathrm{FEV}_{1}$ values were within $5 \%$. Normal saline was then admininstered for 30 seconds and the $F E V_{1}$ recorded once after 30 seconds and repeated only if technically unsatisfactory. The nebuliser chamber was then emptied and dried before being primed with the lowest concentration of histamine, which was given $30 \mathrm{sec}-$ onds later. The procedure was repeated with succes-

Dose regimen for Yan method

\begin{tabular}{|c|c|c|c|c|c|c|c|c|c|}
\hline Dose number & 1 & 2 & 3 & 4 & 5 & 6 & 7 & 8 & 9 \\
\hline $\begin{array}{l}\text { Nebuliser concentration }(\mathrm{g} / 100 \mathrm{ml}) \\
\text { Number of puffs } \\
\text { Cumulative dose histamine }(\mu \mathrm{mol})\end{array}$ & $\begin{array}{l}0.3 \\
1 \\
0.03\end{array}$ & $\begin{array}{l}0.3 \\
1 \\
0.06\end{array}$ & $\begin{array}{l}0.6 \\
1 \\
0.12\end{array}$ & $\begin{array}{l}0.6 \\
2 \\
0.24\end{array}$ & $\begin{array}{l}2.5 \\
1 \\
0.49\end{array}$ & $\begin{array}{l}2.5 \\
2.98 \\
0.98\end{array}$ & $\begin{array}{l}2.5 \\
4 \\
1.8\end{array}$ & $\begin{array}{l}5 \\
4 \\
3.9\end{array}$ & $\begin{array}{l}5 \\
8 \\
7.8\end{array}$ \\
\hline
\end{tabular}




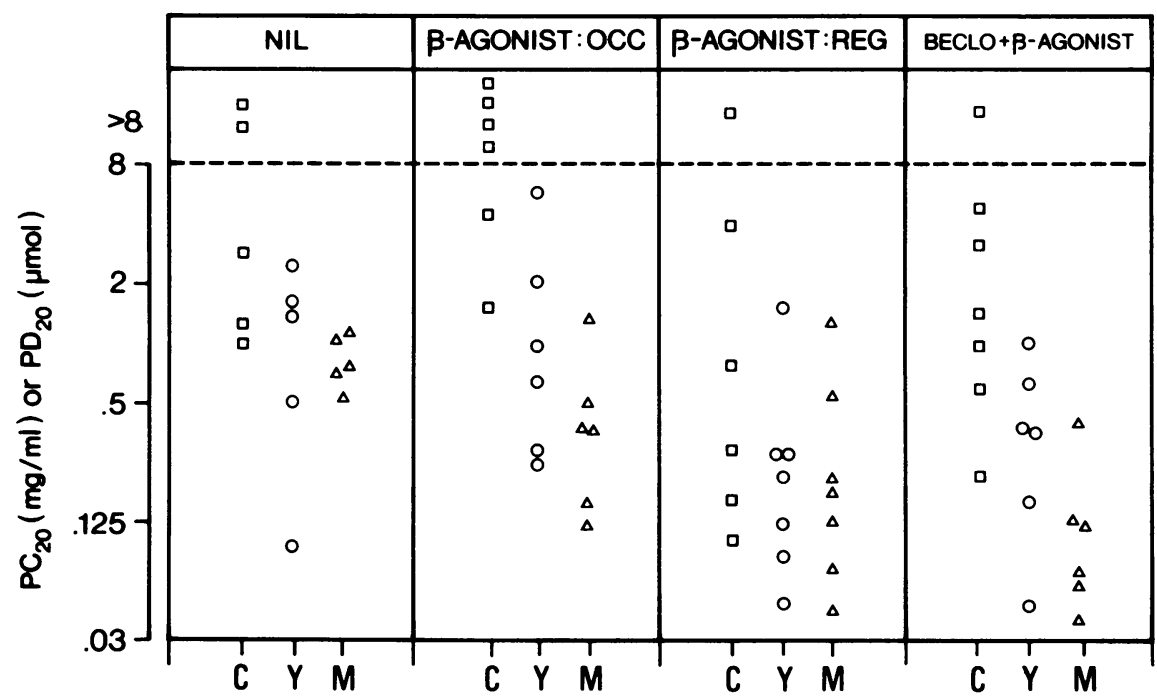

Fig 1 Mean of duplicate $P C_{20}(\mathrm{mg} / \mathrm{ml})$ and $P D_{20}$ ( $\left.\mu \mathrm{mol}\right)$ values from the Cockcroft $(C)$, Yan ( $Y$ ), and Mortagy (M) methods for all subjects according to the medication in use at the time of the study: no medication (NIL), $\beta$ agonist occasionally but less than once a day ( $\beta$-AGONIST:OCC), $\beta$ agonist regularly at least once a day ( $\beta$-AGONIST:REG), and daily $\beta$ agonist plus beclomethasone (BECLO $+\beta-A G O N I S T)$.

sive histamine solutions, in doubling concentrations from 0.3 to $8 \mathrm{mg} / \mathrm{ml}$, at 90 second intervals.

\section{ANALYSIS OF RESULTS}

The percentage fall in $\mathrm{FEV}_{1}$ from the postsaline value was plotted against log dose of histamine. The concentration (Cockcroft and Mortagy methods) or cumulative dose (Yan method) of histamine that provoked a $20 \%$ fall in $F V_{1} \quad\left(P_{20}\right.$ and $P_{20}$ respectively) was then estimated by linear inter- polation.

$\log _{10}$ transformations of $\mathrm{PC}_{20}$ and $\mathrm{PD}_{20}$ values were used in all calculations. The differences between pairs of measurements for each method were tested for normality by means of the modified KolmogorovSmirnov test, ${ }^{6}$ and examined to ensure that the difference between measurements was independent of the magnitude of the measurements (homoscedasticity) by correlating the difference between pairs with their mean value.?

$\mathrm{PC}_{20}(\mathrm{mg} / \mathrm{ml})(2)$

$\mathrm{PD}_{20}(\mu \mathrm{mol})(2)$

$\mathrm{PC}_{20}(\mathrm{mg} / \mathrm{ml})(2)$
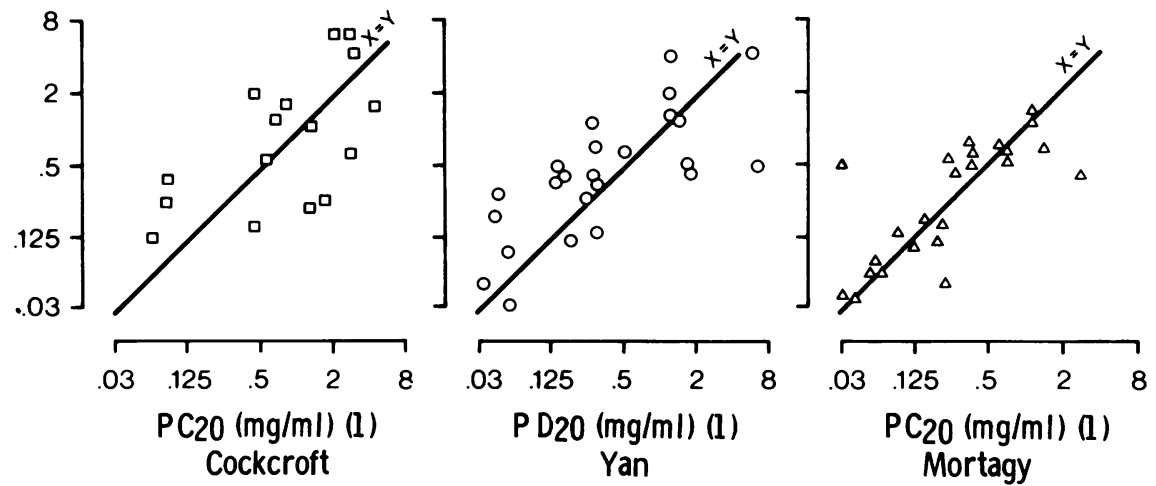

Fig 2 Comparison of the first estimate (1) of $P C_{20}$ and $P D_{20}$ values for each method against second estimate (2) in subjects in whom two estimates were obtained. 
The standard deviation of the differences between measurements was used to construct $95 \%$ ranges for a single measurement by each method from the formula $t_{0.05}(\mathrm{SD}) / \sqrt{2}$. Repeatability was compared by the $\mathrm{F}$ ratio and by Friedman's test.

\section{Results}

Geometric mean $\mathrm{PC}_{20}$ and $\mathrm{PD}_{20}$ values for the three methods for subjects classified according to their regular requirements for inhaled medication are shown in figure 1 . Subjects using no medication were seasonal asthmatics and were symptom free at the time of study.

With the Yan and Mortagy methods $\mathrm{PD}_{20}$ or $\mathrm{PC}_{20}$ values were obtained on both occasions in all 24 subjects. With the Cockcroft method a $\mathrm{PC}_{20}$ of up to $8 \mathrm{mg} / \mathrm{ml}$ was achieved on both occasions in 16 subjects, on one occasion in four, and on neither occasion in four subjects.

For the subjects in whom two $\mathrm{PC}_{20}$ or $\mathrm{PD}_{20}$ values were obtained mean values with the Cockcroft technique were higher than with the Mortagy method by an average of 2.30 doubling concentrations, and numerically higher (in $\mathrm{mg} / \mathrm{ml}$ ) than $\mathbf{P D}_{20}$ values from the Yan method (in $\mu \mathrm{mol}$ ) by a factor of 1.75 doubling increments.

The mean differences (SD in parentheses) between repeat measurements for the Cockcroft, Yan, and Mortagy methods in the 16 subjects in whom both measurements were within the administered dose range were $-0.03(0.49),-0.11(0.43)$, and -0.06 (0.40) $\log _{10}$ units (fig 2). The differences were normally distributed for the Cockcroft and Yan methods, but not for the Mortagy method. Use of parametric statistical methods, however, did not indicate significant bias between first and second readings with each method (paired $t$ test) or any difference in variance between methods ( $F$ ratio). Non-parametric comparison of repeatability (by Friedman's test) also showed no difference between methods. The $95 \%$ ranges for a single measurement for the Cockcroft, Yan, and Mortagy methods were $\pm 2.40,2.11$, and 1.94 doubling concentrations or doubling doses.

\section{Discussion}

The purpose of this study was to select a challenge method for a large community study of asthma prevalence, for which the Cockcroft method was considered too slow and cumbersome to be practical. We therefore compared the Cockcroft method with two methods that had already been applied successfully in epidemiological work. The main criterion was the repeatability of the three methods we were comparing.
The difference between repeated measurements was normally distributed for the Cockcroft and Yan methods, but non-normal for the Mortagy method. We compared repeatability using the parametric $F$ statistic, as appropriate for the Yan and Cockcroft method, on the grounds that this test will if anything overestimate the chances of a difference between the Mortagy and the other two methods. Despite this, there was no difference in repeatability between the three techniques.

The repeatability of bronchial reactivity measurements depends on both subject and measurement factors - that is, the variability in the patients' nonspecific reactivity between tests and the consistency of the technique used to measure reactivity. By comparing the three methods in the same patients we are assuming that biological variability will be similar between the two measurements with each technique, so that the comparison of the three techniques is valid. These assumptions cannot, however, be made when we are comparing results from different groups of subjects. Subjects selected because their asthma is stable or who have previous experience of challenge testing would inevitably show better repeatability than less stable or less experienced asthmatic patients. This is likely to be the explanation for the less good repeatability for the Cockcroft method in our study than in some previous reports. ${ }^{8-12}$ For most of the subjects in our study this was the first experience of both spirometry and histamine challenge, and in this respect they are representative of subjects encountered in epidemiological studies.

The $\mathbf{P C}_{20}$ and $\mathbf{P D}_{20}$ values in our study show a similar relationship to symptoms of asthma to that seen in previous studies for all three methods - up to $20 \mathrm{mg} / \mathrm{ml},{ }^{13} 1 \mu \mathrm{mol},{ }^{3}$ and $0.5 \mathrm{mg} / \mathrm{ml}^{4}$ in asthmatic subjects with symptoms for the Cockcroft, Yan, and Mortagy methods respectively and up to $60 \mathrm{mg} / \mathrm{ml}^{13}$ and $5 \mu \mathrm{mol}^{3}$ in symptom free asthmatics for the Cockcroft and Yan methods.

The failure to demonstrate a $\mathbf{P C}_{20}$ in all subjects with the Cockcroft technique is presumably due to a lower maximum inhaled dose of histamine. The total dose of histamine emitted from the nebuliser, 14.6 $\mu \mathrm{mol}$, is delivered continuously during inspiration and expiration so most of it is lost to the atmosphere. In contrast, the maximum doses of 7.8 and $7.3 \mu \mathrm{mol}$ with the Yan and Mortagy methods are delivered during inspiration only. These two methods were also completed in a shorter time, so a greater proportion of the cumulative histamine dose would be active at the end of the protocol.

Both the Yan and the Mortagy protocols were easy to perform, though the need to clean the nebuliser chamber between doses with the Mortagy method occupied most of the time available. A further disad- 
vantage of the Mortagy technique is that the output of the Pulmasonic nebuliser is technically difficult to measure, so that proper standardisation is difficult. Thus, of the three methods tested, the Yan technique was the simplest. It is fast, convenient, and inexpensive compared with the Cockcroft method, and in a clinical setting did not compromise repeatability. These qualities offer potential advantages for clinical and epidemiological use.

We are grateful to Dr DJ Lipscomb at Basingstoke Hospital for permission to approach his patients for the study, to Miss Susan Chinn for statistical advice, and to Mrs M Dowling and Mrs J Galletti for secretarial assistance.

\section{Addendum}

In an abstract of this work presented at the British Thoracic Society meeting in July 1984 (Thorax 1984;39:698), repeatability of method was expressed from the repeatability coefficient, ${ }^{6}$ with values of 4.43 , 4.00, and 3.67 doubling concentrations for the Cockcroft, Yan, and Mortagy methods respectively. The 95\% ranges quoted in this paper have been substituted for ease of comparison with other studies.

\section{References}

1 Tiffeneau R. Hypersensibilité cholinergo-histaminique pulmonaire de l'asthmatique. Acta Allergol 1958;suppl V:187-221.

2 Cockcroft DW, Killian DN, Mellon JJA, Hargreave FE. Bronchial reactivity to inhaled histamine: a method and clinical survey. Clin Allergy 1977;7:235-43.

3 Yan K, Salome C, Woolcock AJ. A rapid method for measurement of bronchial responsiveness. Thorax 1983;38:760-5.

4 Mortagy AK. Respiratory symptoms and bronchial reactivity: studies on their prevalence and clinical significance. $\mathrm{PhD}$ thesis, University of Southampton, 1984.

5 Cotes JE. Lung function: assessment and measurement. 3rd ed. Oxford: Blackwell Scientific Publications, 1975:366-7.

6 Strike PW. Medical laboratory statistics. 1st ed. Bristol: John Wright, 1981:47-50.

7 Altman DG, Bland JM. Measurement in medicine: the analysis of method comparison studies. The Statistician 1983;32:307-17.

8 Juniper EF, Syty-Golda M, Hargreave FE. Histamine inhalation tests: inhalation of aerosol via a face mask or a valve box with mouthpiece. Thorax 1984;39:556-7.

9 Ryan G, Dolovich MB, Roberts RS, et al. Standardisation of inhalation provocation tests: two techniques of aerosol generation and inhalation compared. Am Rev Respir Dis 1981;123:195-9.

10 Juniper EF, Frith PA, Dunnett C, Cockcroft DW, Hargreave FE. Reproducibility and comparison of responses to inhaled histamine and methacholine. Thorax 1978;33:705-10.

11 Cockcroft DW, Berscheid BA, Murdock KY. Measurement of responsiveness to inhaled histamine using FEV 1 : comparison of $\mathrm{PC}_{20}$ and threshold. Thorax 1983;38:523-6.

12 Dehaut P, Rachiele A, Martin RR, Malo JL. Histamine dose-response curves in asthma: reproducibility and sensitivity of different indices to assess response. Thorax 1983;38:516-22.

13 Ryan G, Latimer KM, Dolovich J, Hargreave FE. Bronchial responsiveness to histamine: relationship to diurnal variation of peak flow rate, improvement after bronchodilator, and airway calibre. Thorax 1982; 37:423-9. 